\title{
Study of Reduced System Models for Power System Monitoring
}

\author{
${ }^{* 1}$ A Kranthi Kumar, ${ }^{2}$ Ch. V. V. S. Bhaskara Reddy \\ Department of Electrical Engineering, $\operatorname{AUCE}(\mathrm{A})$, Visakhapatnam, India \\ Email: kranthikumar.aradhadi@gmail.com
}

\section{Received: 10 ${ }^{\text {th }}$ December 2017, Accepted: 17th January 2018, Published: 28th February2018}

\begin{abstract}
With increase in loads and generation the complexity and interconnection of power system are increasing. It necessitates more computational time to study the system behaviour for large power systems. During the emergency conditions like faults the computational time must be less. The network reduction techniques are useful in analysing large interconnected systems with less computational time. Some of the network reduction techniques, such as Ward reduction, Kron reduction and Dimo's method are discussed in this paper. The effectiveness of these reduced models are analysed with their ability to follow the bus voltages of the complete system. The IEEE 14 bus and 118 bus systems are taken as the test cases.
\end{abstract}

Keywords: Dimo's method, Ward reduction, Kron reduction, L-index, VCPI.

\section{Introduction}

Modern power systems have become highly interconnected to maintain the quality and reliability of the supply. These systems are being operated under heavily loaded condition to meet the growing demand for electricity with limited resources. Over the past few decades, the characteristics of the power system generation and loads have been changed rapidly due to increase in renewable energy sources, frequent usage of power electronic devices. Therefore, special attention has to be given for the modelling of power system for various types of analysis.

In a power system it is difficult or not economical to develop a detailed model although we have powerful computing capabilities available nowadays. Therefore, it is necessary to develop the reduced models that properly resemble the detailed model for steady state and dynamic characteristics. The reasons for using the reduced models are as follows: (i) it is required to monitor the system by using limited number of measurements from measuring units, such as PMU's; (ii) in the interconnected systems they refuse to share the information with the others; (iii) practical limitations on the computational resources; (iv) as the electrical distance from the point of interest increases, the requirement of detailed model decreases.

Power systems are mostly interconnected, with several areas connected with each other by tie-lines. Usually the utilities of own system are called internal system or internal area and the rest of the system is called external system for internal area considered. For power system analysis the internal area is modelled in detail and external areas are represented by simplified models, which are referred as the external equivalent systems. The problem of network reduction is not only related to the intercompany ties but, some of the individual systems are grown to such an extent that it represents its own power system.

Network reduction techniques are broadly classified as static and dynamic based on its intended use [1-2]. Static reduction techniques are used for static performance analysis only, such as load-flow analysis and system operation and planning studies. Whereas, for the analysis of system dynamics the reduced dynamic equivalents of the external system are required. In this paper, the term 'network reduction' implies only the static reduction.

This paper compares the effectiveness of some of the commonly used network reduction methods, such as Ward reduction, Kron reduction and Dimo's method.

\section{Materials and Methods}

A. Ward reduction:

The construction of ward equivalents starts from the solved model of the entire power system. The current injection $I(i)$ at each bus $\mathrm{i}$ is obtained from the known bus voltage $V(i)$ and complex power $S(i)$

$$
I(i)=S^{*}(i) / V^{*}(i)
$$

let a power system can be represented by the nodal equation,

$$
Y_{\text {Bus }} V=i
$$

Where $\mathrm{Y}_{\mathrm{Bus}}$ is the admittance matrix of size $n x n, \mathrm{~V}$ is the complex voltage vector of size $n \times 1$ and $I$ is the complex current vector of size $n \times 1, n$ is the number of nodes or buses in the system. The reduction of admittance matrix and the current vector is performed by using Gaussian elimination technique. let the network having $\mathrm{n}$ node $\mathrm{s}$ can be divided into retained nodes $\{R\}$ and eliminated nodes $\{E\}$ as shown in the Fig. (1). 


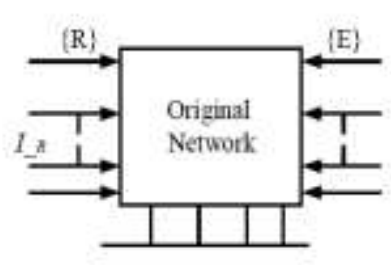

(a)

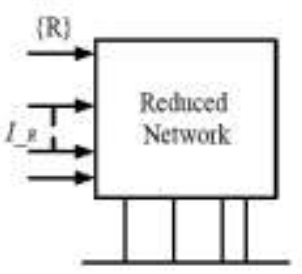

(b)
Fig.1 (a)Original network and (b)Reduced network vided into retained nodes $\{\mathrm{R}\}$ and eliminated nodes $\{\mathrm{E}\}$ as shown in the Fig. (1).

After the elimination of kth node, the $\mathrm{Y}_{\mathrm{Bus}}$ is modified as,

$$
Y_{i j}^{\prime}=Y_{i j}-\frac{Y_{i k} Y_{j k}}{Y_{k k}} ; \mathrm{i}, \mathrm{j}=1,2, \ldots ., \mathrm{n} ; \mathrm{i}, \mathrm{j} \neq \mathrm{k}
$$

Similarly, after elimination of kth node the current vector is modified as,

$$
I^{\prime}(i)=I(i)-\frac{Y_{i k}}{Y_{k k}} I(i)
$$

The element $\mathrm{Y}_{\mathrm{ij}}$ ' distinguishes the elements of the new (n-1)x(n-1) $Y_{\text {Bus }}$ from the original nxn $Y_{\text {Bus, }}$, and I' is the modified current injection vector. The Gaussian elimination method will eliminate one node at a time sequentially removed from the original system. If the network is reduced to $\mathrm{r}$ nodes, the dimension of new admittance matrix is rxr and dimension of new current injection vector is rx1. After the reduction, the new current vector is converted back to complex power with Eq. (1) and it is used for the load-flow solutions [3-5]. This reduced network carries the full information of the original power system at the base case.

\section{B. Kron reduction:}

The Gaussian elimination method removes the need of matrix inversion for solving nodal equations of a large-scale power system. it is also shown that elimination of variables is identical to the network reduction since it leads to reduced order network equivalents by node elimination at each step. This is used analyze the large interconnected power system if there is special interest in voltages at some buses of the system.

current injection is always zero at those buses which have no connected load or generators and voltages at these buses need not be calculated and they are eliminated. the current injection at the non-essential nodes converted to a constant shunt admittance value by

$$
Y_{k k}=-\left(P_{L k}-j \cdot Q_{L k}\right) / \bar{V}_{k}^{2}
$$

where $P_{L K}$ and $Q_{L k}$ are injected active and reactive loads at the kth bus, these shunt admittances are added to the $Y_{\text {Bus }}$ diagonal elements at the respective buses. Now the equivalents become passive network with zero current injections. The admittance bus elimination and current injection vector is modified by Eq. (3) and Eq. (4).
At these buses we need not calculate the voltages and they can be eliminated. these equivalents can be constructed from previously calculated or assumed bus power and voltage magnitudes [5-6].

\section{Dimo's Reduction:}

Dimo equivalent is to aggregate the power and the current injection of the buses which are to be eliminated and connected to a fictitious ground through impedances while preserving the properties and the power balance of the base case. The fictitious ground is connected to the fictitious load center through impedance. This method is known as the "REI equivalencing" and steps in the process of building zero power balance network are

1. Define the set of nodes to be retained and to be aggregated.

2. Analyse the nodal injections at the nonessential buses and define the aggregating pattern for generations and load.

3. Assemble ZPBN.

4. Assemble bus admittance matrix for extended model.

5. Gaussian elimination of non-essential buses.

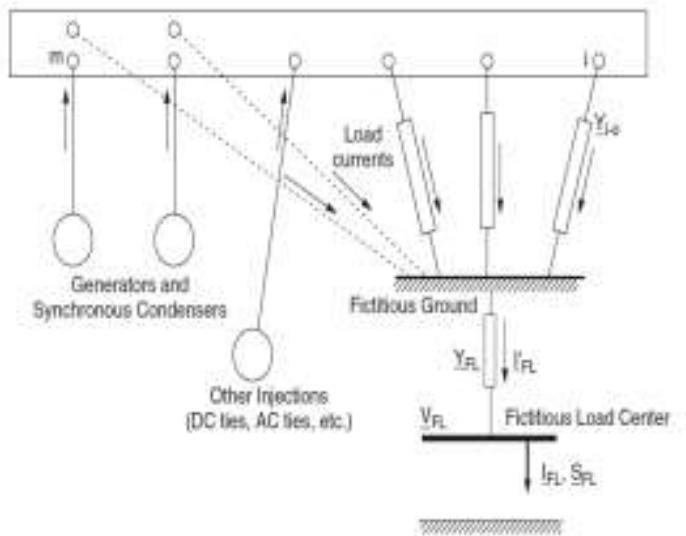

Fig. 2 Zero power Balance Network

all the non-essential nodes are replaced by linearized impedances, and are connected to the fictitious ground. The impedances are calculated as

$$
\begin{aligned}
& B(i)=-Q(i) / V^{2}(i) \\
& G(i)=P(i) / V^{2}(i)
\end{aligned}
$$

where $B(i)$ and $G(i)$ are the susceptance and conductance between the $i^{\text {th }}$ non-essential node and fictitious ground respectively. $P(i), Q(i)$ and $V(i)$ are active power, reactive power and complex voltage at the $\mathrm{i}^{\text {th }}$ non-essential bus respectively.

The current $i_{\text {Ag }}(i)$ from these non-essential nodes (A) to the fictitious ground $(\mathrm{g})$ is calculated as

$$
i_{A g}(i)=\left(S_{A}(i) / V_{A}(i)\right)^{*}
$$


and the current between fictitious ground and fictitious load center ( $f$ ) is the sum of current flowing from non-essential buses into the fictitious ground,

$$
\bar{I}_{g f}=\sum_{i \in\{A\}} i_{A g}(i)
$$

application of the power conservation theorem at the fictitious ground gives

$$
\bar{S}_{g f}=\sum_{i \in\{A\}} S_{A g}(i)
$$

with the knowledge of complex power and current flowing into the fictitious load center are use $d$ calculate the line parameters $(R$ and $X)$, between the nodes

$$
R+j \cdot X=\bar{S}_{g f} / \bar{I}_{g f}^{2}
$$

Replacing the loads in the original system with the ZPBN produces an extended network that has exactly the same power balance as the original network [7-8].

The complex voltage at the fictitious load center is calculated as

$$
V_{f}=\bar{S}_{g f} / \bar{I}_{g f}^{*}
$$

the REI net is obtained by applying short circuit transformation, that is by applying Gaussian elimination to all the passive buses in the extended network. After the elimination we only retain the generator buses and the fictitious load bus. another different type of reduction, instead of expanding the original network with ZNPB, by replacing the loads with impedances except for the bus we want study. After Gaussian elimination we only retain the generator buses and load bus we want to study.

\section{Voltage stability indices:}

Voltage stability indices define the status and condition of the power system stability with respect to voltage stability. The distance between actual state and voltage collapse point is known by defining critical buses of the system. Some of the indices are discussed in this paper.

\section{L-index:}

Kessel et.al [9] has derived voltage stability index based on power flow solution. Using this index, we will know how far the actual system from the collapse point is. The L-index describes the stability of a system.

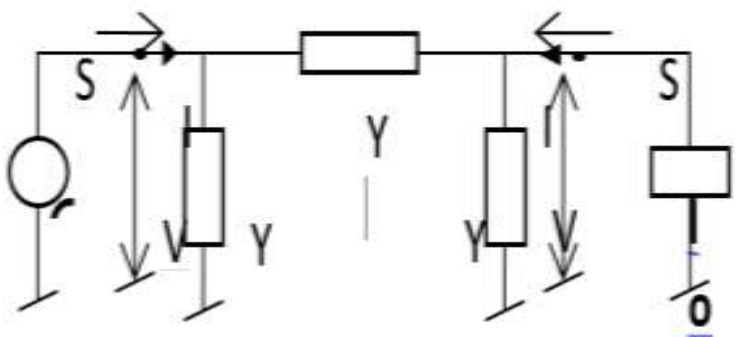

Fig. 3 Line model

Consider a network with ' $n$ ' node consists of consumer node (PQ node) and generator nodes (PV nodes). The relation between voltages and currents can be represented using hybrid matrix $\mathrm{H}$ given by:

$$
\left|\begin{array}{l}
V^{L} \\
I^{G}
\end{array}\right|=|H| \cdot\left|\begin{array}{l}
I^{L} \\
{ }_{V} G
\end{array}\right|=\| \begin{aligned}
& Z^{L L} \| F^{L G} \\
& K_{K}^{G L} \|{ }_{Y} G G \mid
\end{aligned}|\cdot| \begin{aligned}
& I^{L} \\
& { }_{V} G
\end{aligned} \mid
$$

Where,

$V^{L}, I^{L}$ Vectors of voltages and currents at consumer nodes

${ }_{V}^{G},{ }^{G}$ Vectors of voltages and currents at generators

$$
Z^{L L}, F^{L G}
$$$$
K^{G L}, Y^{G G}
$$

Submatrices of the H-matrix The L Index is given by

$$
L=\underset{j \in \alpha_{L}}{\operatorname{MAX}}\left|1-\frac{\sum_{i \in \alpha_{G}} F_{j i} \cdot V_{i}}{V_{j}}\right|<1
$$

Where,

$\mathrm{V}_{\mathrm{i}}$ : Voltage at generator bus

$\mathrm{V}_{\mathrm{j}}$ : Voltage at load bus.

Therefore, for stability $L<1$ is a must condition.

Voltage Collapse Proximity Indicator:

Using maximum power transfer theory of a line by Chebbo et al. [10] derived a $\mathrm{Z}$ ratio model. Consider a two bus system Which consists of constant voltage source with internal impedance of $\mathrm{Z}_{s}$ and a load impedance of $\mathrm{Z}_{l}$. According to maximum power transfer theorem maximum power will be delivered from source to load when $\mathrm{Z}_{s}=\mathrm{Z}_{l}$ i.e., $Z_{s} / Z_{l}=1$

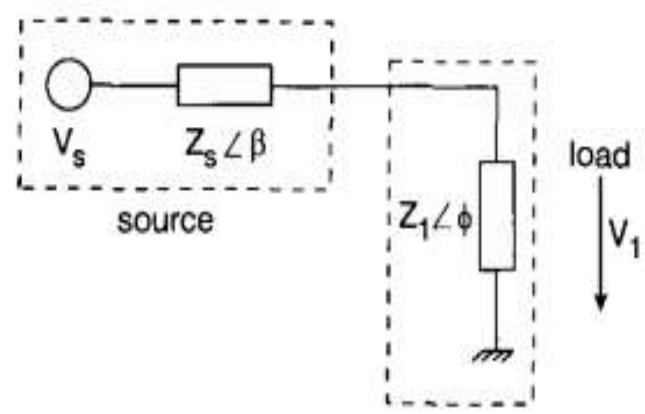


Fig. 4 Two Bus system

When this condition is generalized for $\mathrm{n}$ bus system this can be used as a voltage collapse proximity indicator. Therefore the Thevenin's equivalent impedance between bus $\mathrm{i}$ and ground is $\mathrm{Z}_{l l} \angle \beta_{l}$, and $Z_{i} \angle \phi_{i}$.

Where,

$Z_{i} \angle \phi_{i}$ is the load impedance $Z_{i i} \angle \beta_{i}$ is the $i^{\text {th }}$ diagonal element of $[Z]=[Y]^{-1}$. Therefore a permissible power is supplied to a particular bus $i$ is possible when the ratio $Z_{i i} / Z_{i}$ is less than or equal to one. Thus for stable and secure operation of a system

$$
Z_{i i} / Z_{i} \leq 1
$$

\section{Case Study}

The equivalents discussed in the last section were used IEEE 14 bus system and IEEE 118 bus system. All these tests are based on the initial load flow solution of the complete system. Sensitive buses in the system are identified from the calculations of Lindex and VCPI. All the external buses are eliminated in the reduction techniques. Load flow analysis is carried out on complete and reduced system with a set of percentage change in loads and corresponding generation rescheduling.

\section{A. IEEE 14 Bus System}

The L-index and VCPI are calculated for the complete system and shown in Table I. From the results of L-index and VCPI the sensitive nodes in the system are identified and now we can retain those weak buses in the internal system along with generator buses and we can treat all other buses as the external system buses. The division of internal and external buses is shown in the Table II. The external system buses are eliminated using the network reduction techniques discussed earlier.

Table I. L-index and VCPI for IEEE 14 System

\begin{tabular}{|c|c|c|}
\hline Bus No & L-index & VCPI \\
\hline 4 & 0.0298 & 0.0485 \\
\hline 5 & 0.0203 & 0.0337 \\
\hline 7 & 0 & 0 \\
\hline 9 & 0.0675 & 0.1931 \\
\hline 10 & 0.0644 & 0.1004 \\
\hline 11 & 0.0364 & 0.0525 \\
\hline 12 & 0.0240 & 0.0693 \\
\hline 13 & 0.0320 & 0.1323 \\
\hline 14 & 0.0780 & 0.1212 \\
\hline
\end{tabular}

Table II. Partition of IEEE 14 System

\begin{tabular}{|c|c|}
\hline Internal System Buses & External System Buses \\
\hline $1,2,3,6,8,9,10,14$ & $4,5,7,11,12,13$ \\
\hline
\end{tabular}

To test the effectiveness of these reduction techniques, the load is varied by $\pm 5 \%, \pm 10 \%, \pm 15 \%$ and the generations are rescheduled accordingly. In Fig.5 voltage magnitudes of the some of the internal buses are plotted and compared for variation in load of entire power system to variation in only the internal system. Full Newton-Raphson load-flow technique is used for all reduction techniques. It is observed that with the increase or decrease in load, the per unit p.u. error increased at the boundary buses. With the highest error being at the bus number 14 at $15 \%$ increase in load. The maximum error observed is 0.0199 p.u. in case of Kron reduction. The maximum error for Dimo's reduction technique is 0.0098 . The least error is obtained using Ward reduction, i.e., 0.0014 .

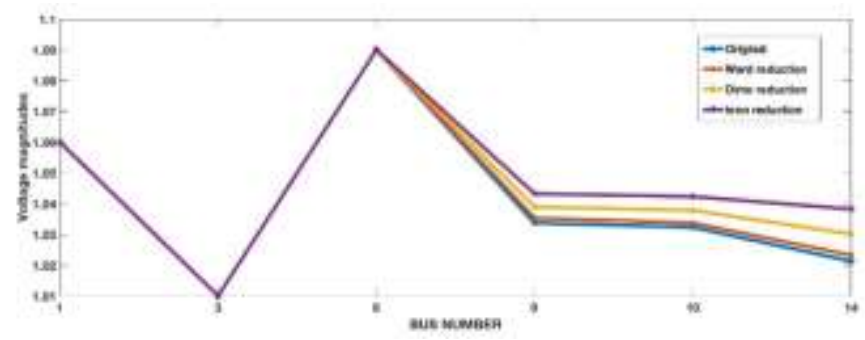

5. (a) Base case

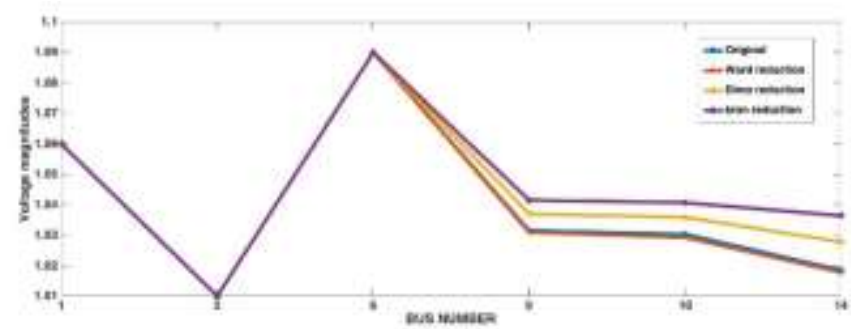

5. (b) increase in $5 \%$ of load

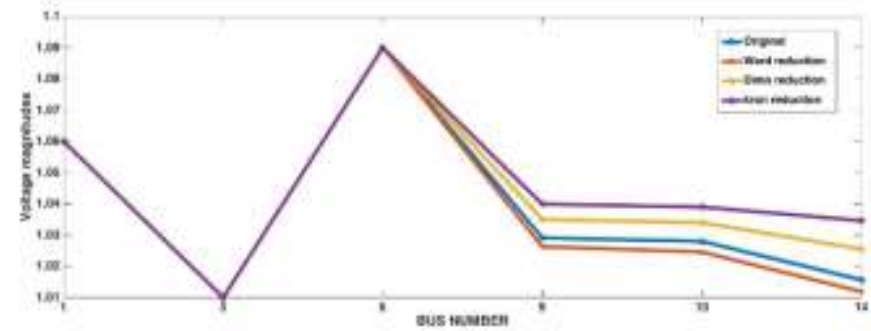

5. (c) Increase in $10 \%$ of load 


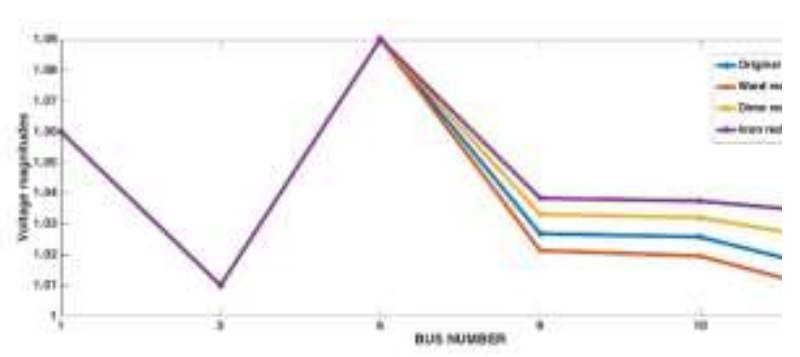

5. (d) Increase in $15 \%$ of load

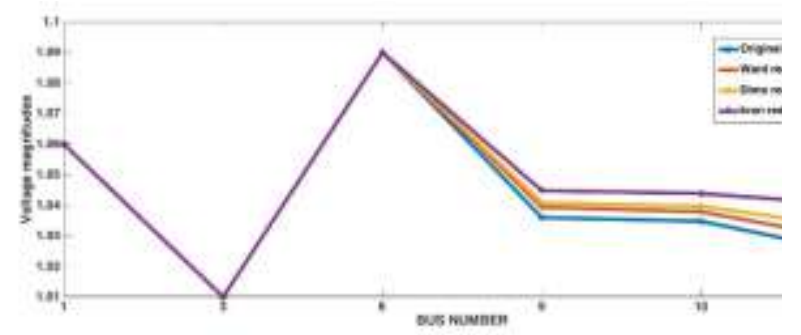

5. (e) Decrease in $5 \%$ of load

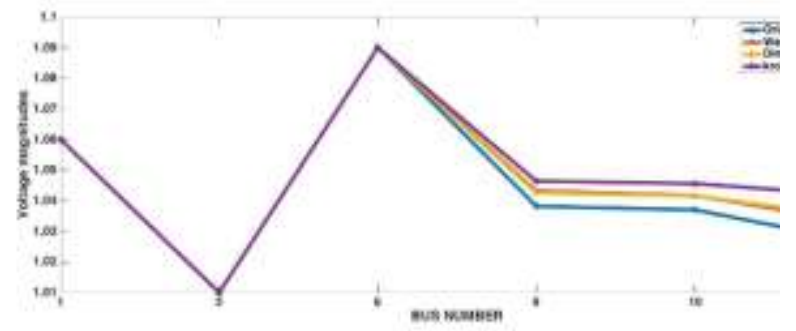

5. (f) Decrease in $10 \%$ of load

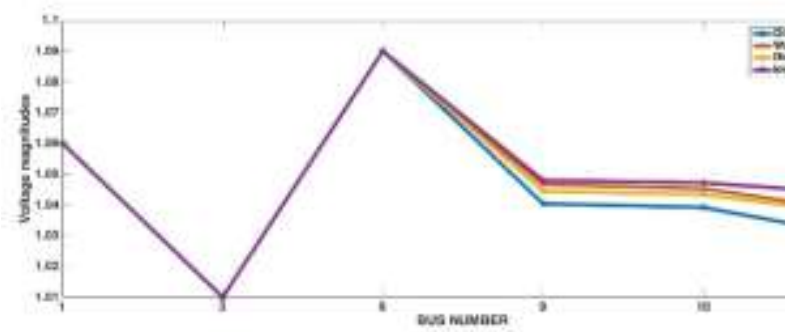

5. (g) Decrease in $15 \%$ of load

Fig. 5 Variation of retained bus voltages magnitude with change in load for IEEE 14 bus system

\section{B. IEEE 118 Bus system}

The L-index and VCPI for complete system are calculated and from the results of L-index and VCPI the sensitive nodes in the system are identified and now we can retain those weak buses in the internal system. The division of internal and external buses is done for the IEEE 118 Bus System.

Table. III L-index and VCPI for IEEE 118 system For sensitive buses

\begin{tabular}{|c|c|c|}
\hline Bus No & L-index & VCPI \\
\hline 21 & 0.0489 & 0.0999 \\
\hline 22 & 0.0409 & 0.0909 \\
\hline 43 & 0.0542 & 0.1042 \\
\hline 44 & 0.0774 & 0.1277 \\
\hline 45 & 0.0650 & 0.1134 \\
\hline 52 & 0.0495 & 0.0995 \\
\hline
\end{tabular}

\begin{tabular}{|l|l|l|}
\hline 82 & 0.0501 & 0.1030 \\
\hline 83 & 0.0438 & 0.0984 \\
\hline
\end{tabular}

In the Table III the retained load buses are shown. The load is varied by $\pm 5 \%, \pm 10 \%, \pm 15 \%$ and the voltage magnitude at the retained node are observed. In Fig.6 voltage magnitudes of the some of the internal buses are plotted and compared for variation in load of entire power system to variation in only the internal system. It is observed that the same trend is followed in the IEEE 118 bus system case also that is with the increase or decrease in load, the per unit p.u. error increased at the boundary buses. With the highest error being at the bus number 82 at $15 \%$ increase in load. The maximum error observed is 0.0108 p.u. in case of Kron reduction. The maximum error for Dimo's reduction technique is 0.0093 . The least error is obtained using Ward reduction, i.e., 0.0084 . The maximum error in p.u. for different methods are represented in Table IV.

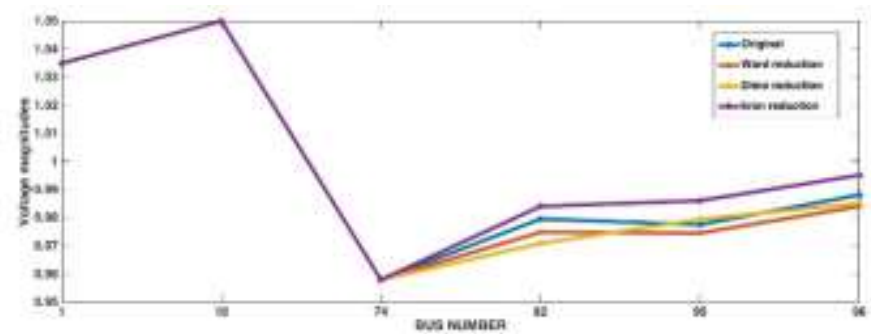

6. (a) Base case

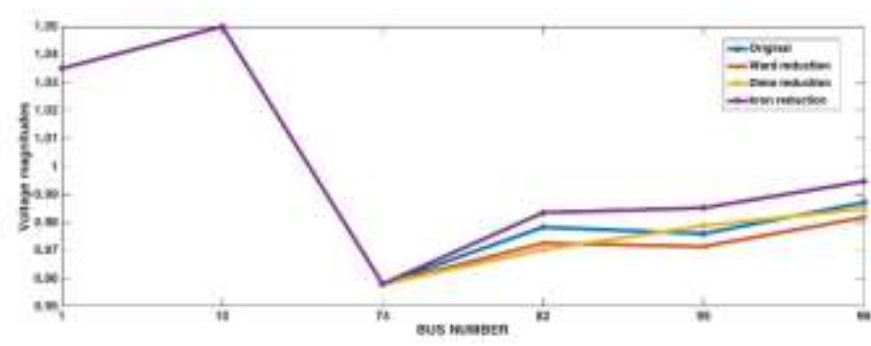

6. (b) Increase in $5 \%$ of load

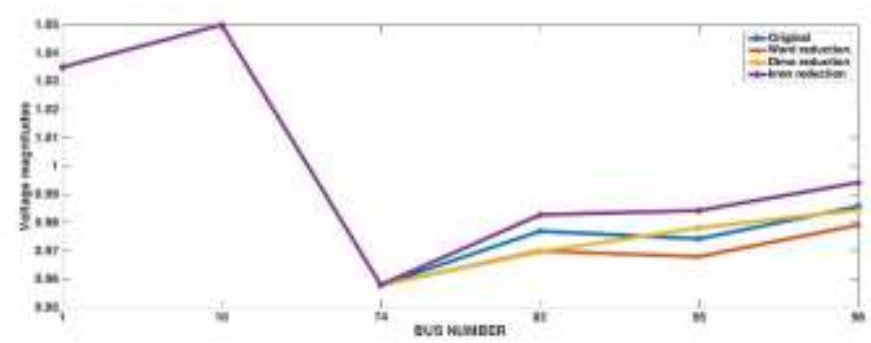

6. (c) Increase in $10 \%$ of load 


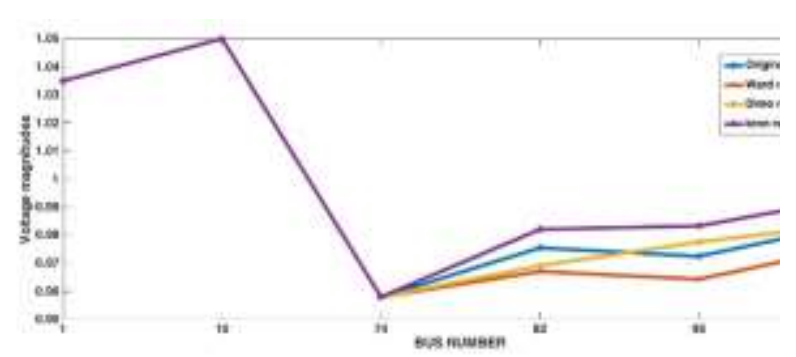

6. (d) Increase in $15 \%$ of load

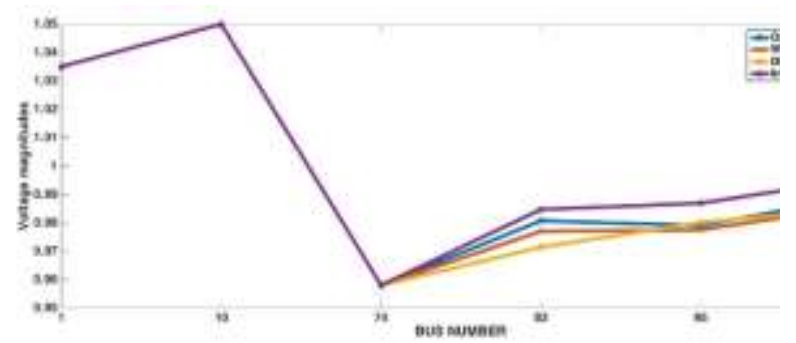

6. (e) Decrease in $5 \%$ of load

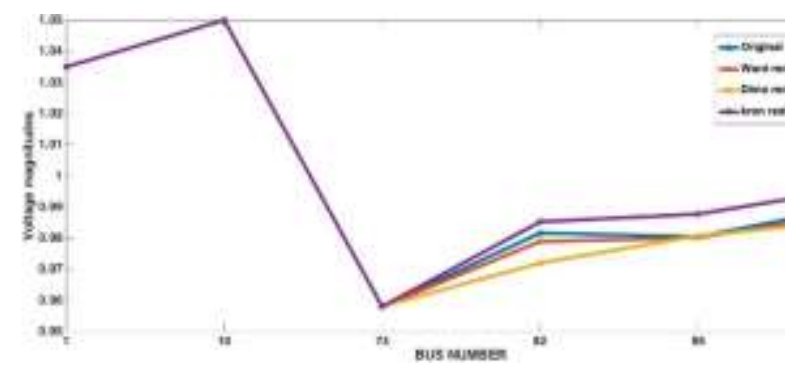

6. (f) Decrease in $10 \%$ of load

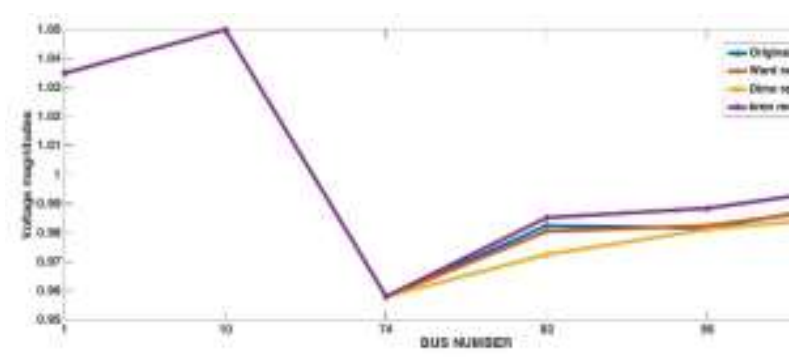

6. (g) Decrease in $15 \%$ of load

Fig. 6 Variation of retained bus voltages magnitude with change in load for IEEE 118 bus system

Table. IV Error comparison for IEEE 14 and 118

\begin{tabular}{|c|c|c|}
\hline \multicolumn{3}{|c}{} \\
$\begin{array}{c}\text { Reduction } \\
\text { Techniques }\end{array}$ & $\begin{array}{c}\text { beEE } 14 \text { Bus } \\
\text { System }\end{array}$ & $\begin{array}{c}\text { IEEE 118 Bus } \\
\text { System }\end{array}$ \\
\cline { 2 - 3 } & Max error & Max error \\
\hline Ward & 0.0071 & 0.0084 \\
\hline Kron & 0.0199 & 0.0108 \\
\hline Dimo & 0.0098 & 0.0093 \\
\hline
\end{tabular}

\section{Conclusion}

Network reduction methods are found to be very useful in the analysis of very large and complex interconnected power systems. IEEE 14 and IEEE
118 bus test systems are analysed and error in voltage magnitude for each method are observed. The accuracy of the Kron method is low as compared to other two methods analysed. Ward reduction technique exhibiting close convergence for both the test systems. The complexity of ward and Kron method is less compared to the Dimo method as it involves the aggregation of nodes.

\section{References}

1. Jan Machowski, Janusz Bialek, and Jim Bumby. Power System Dynamics: Stability and Control. John Wiley \& Sons, 2011.

2. J. F. Dopazo, G. Irisarri and A. M. Sasson, "Real-time External System Equivalent for On-line Contingency Analysis", IEEE Trans. On Power Apparatus and Systems, vol. PAS-98, no. 6, Nov./Dec. 1979.

3. John J. Grainger, and William D. Stevenson. Power System Analysis. New York: McGraw-Hill, 1994.

4. Ward, J. B., "Equivalent Circuits for Power-Flow Studies", Transactions of the American Institute of Electrical Engineers, vol.68, no.1, pp.373,382, July 1949.

5. Arthur R. Bergen, Power Systems Analysis. Pearson Education India, 2009.

6. S. Deckmann, A. Pizzolante, A. Monticelli, B. Stott, O. Alsac, "Numerical Testing of Power System LoadFlow Equivalents," IEEETrans. on Power Apparatus and Systems, vol.PAS-99, no.6, pp.2292,2300, Nov. 1980.

7. S. C. Savulescu, "Equivalents for Security Analysis of Power Systems," IEEE Trans. on Power Apparatus and Systems, vol.PAS-100, no.5, pp.2672,2682, May 1981.

8. S. C. Savulescu. Real-time Stability Assessment in Modern Power System Control Centers. Vol. 42. John Wiley \& Sons, 2009.

9. P. kessel and H Galvitsch "Estimating the voltage stability of a power system" IEEE Transaction on power delivery, vol. 1, no. 3, pp. 346-354, Jul. 1986.

10. Chebbo M.R. Irving M J H Sterling "Voltage collapse proximity indicator: behaviour and implications" IEEE proceedings on Generation, Transmission 
Helix Vol. 8(2): 3087-3092

and Distribution, vol. 145, pp. 634-640,

Nov. 1998 